\title{
Precise Orbit Determination of BDS MEO Satellites Based on Satellite TT\&C Stations
}

\author{
Kezhao Li, ${ }^{1,2}$ Zhiwei Li, ${ }^{1}$ Lin Chai, ${ }^{3}$ An-min Ding, ${ }^{1}$ Jin-ben Wei, ${ }^{1}$ and Long Li ${ }^{1}$ \\ ${ }^{1}$ Henan Polytechnic University, Jiaozuo, China \\ ${ }^{2}$ Collaborative Innovation Center of BDS Research Application, Zhengzhou, China \\ ${ }^{3}$ Southwest China Research Institute of Electronic Technology, Chengdu, China \\ Correspondence should be addressed to Kezhao Li; kz@hpu.edu.cn
}

Received 15 September 2015; Revised 26 December 2015; Accepted 29 December 2015

Academic Editor: Hikmat Asadov

Copyright (C) 2016 Kezhao Li et al. This is an open access article distributed under the Creative Commons Attribution License, which permits unrestricted use, distribution, and reproduction in any medium, provided the original work is properly cited.

A novel method, which is based on the triple-frequency combination and Space-Based Telemetry, Tracking, and Command (STT\&C) stations, is proposed in this paper. Considering BeiDou Navigation Satellite System (BDS) Geostationary Orbit (GEO) and Inclined Geostationary Orbit (IGSO) satellites as the STT\&C facilities, firstly, we presented the BDS Medium Earth Orbit (MEO) satellites' precise orbit determination scheme based on triple-frequency combination. Then, we gave the sufficient and necessary conditions about the visibility and the coverage rate calculation model of STT\&C to BDS MEO satellite. And then we deduced the model of BDS MEO satellites precise orbit determination based on triple-frequency combination observations. At last, we designed the simulation calculation. The simulation results show that orbit determination of BDS MEO satellite based on STT\&C station can be realized at all times. And most of the simulation period time, under the condition of the dm level orbit determination for GEO/IGSO satellites, the position accuracy of the relative orbit determination is better than $4 \mathrm{~m}$, the horizontal accuracy of the relative orbit determination is within $2.5 \mathrm{~m}$, and the vertical accuracy of the relative orbit determination is less than $3.5 \mathrm{~m}$.

\section{Introduction}

The orbital determination accuracy of Global Navigation Satellite System (GNSS) satellites is a vital factor in GNSS navigation and positioning. Due to the limitation of Chinese geographical cover area, only about thirty percent of orbital period of BDS MEO satellites can be tracked by using ground TT\&C stations or ground differential reference stations of the Chinese's own $[1,2]$. Thus it is very difficult to realize the precise orbit determination for BDS MEO satellites. But fortunately, BDS GEO/IGSO satellites can be determined by the Chinese's own ground stations. The testing broadcast ephemeris error of BDS is better than $1.5 \mathrm{~m}$ currently [3]. Steigenberger et al. analyzed the orbit accuracy on the severaldecimeter level for the GEO and the few-decimeter level for IGSO satellites of BDS could be achieved [4]. Additionally, it is a novel way to get the high and fast accuracy positioning results by using multifrequency carrier phase combination technology. But as we know, carrier phase ambiguity must be firstly resolved in GNSS positioning. According to ambiguity resolution (AR) problems, many scholars have done a lot of research. Vollath et al. [5] described the three-carrier ambiguity resolution (TCAR) method, and de Jonge et al. [6] and Hatch et al. [7] proposed the Cascade Integer Resolution (CIR) method. Both early TCAR and CIR use essentially the same geometry-free bootstrapping procedure. Odijk and Teunissen [8] proposed an analytical closed-form expression for the multifrequency ambiguity dilution precision. Feng $[9,10]$ outlined a general modeling strategy for improved AR and positioning estimation using three or more phase and code ranging signals. Paziewski and Wielgosz [11] presented a method by using frequencies L1/E1 and L5/E5a combination to account for Galileo/GPS intersystem biases in precise satellite positioning. In theory, using real tracking data, combined orbit and clock correction precision, the accuracy of the GEO satellite orbit determination can meet $\mathrm{cm}$ level by using multifrequency carrier phase technology [12]. On the basis of these research results, we proposed a tentative idea, which takes BDS GEO/IGSO satellites as STT\&C stations and can solve the problem of BDS MEO satellite precision 


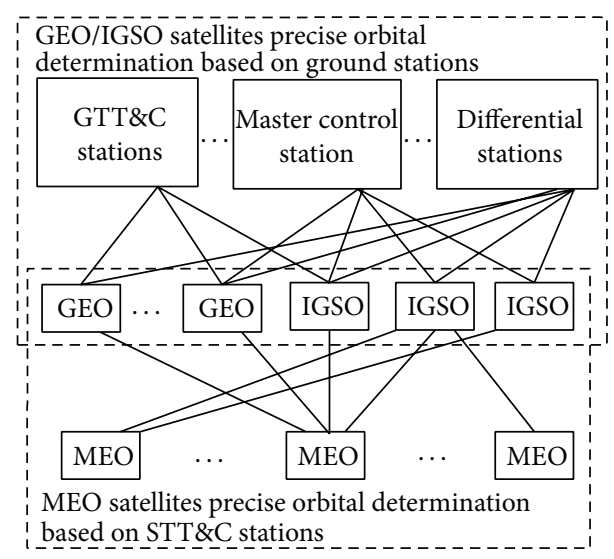

FIGURE 1: The schematic flow diagram of the precise orbit determination of BDS MEO satellites based on STT\&C stations.

orbit determination using multifrequency combination technology.

\section{The Precise Orbit Determination Scheme of BDS MEO Satellites Based on STT\&C}

The precise orbit determination scheme of BDS MEO satellites based on multifrequency combination can be described by the schematic flow diagram in Figure 1.

The scheme of the specific ideas is as follows. (1) All BDS GEO/IGSO satellites can be observed, commanded, and controlled because they are within the range of the ground TT\&C (GTT\&C) stations or differential stations of the Chinese's own. (2) On the basis of the precise orbital determination of $\mathrm{BDS}$ GEO/IGSO satellites, referring to the relative navigation way in Low Earth Orbiting (LEO) by using carrier phase differential GPS [13], we can install the receivers on BDS MEO satellites, which can receive the signal from BDS GEO/IGSO satellites by using omnidirectional antenna. Thus we consider BDS GEO/IGSO satellites as STT\&C stations and can use the method of multifrequency combination technology to realize the precise orbit determination of BDS MEO satellites ulteriorly.

\section{The Visibility and Coverage Rate Calculation Model of STT\&C to BDS MEO Satellite}

Using STT\&C to determine the orbit of BDS MEO satellite, the visibility condition of STT\&C to BDS MEO satellite must be met. The sufficient and necessary conditions about the visibility are deduced. And the coverage rate calculation model about it is given as follows.

3.1. The Description about the Visibility Condition of STT\&C to BDS MEO Satellite. For the BDS MEO satellite is under the GEO/IGSO constellation, the pitch angle $E$ of STT\&C is a minus (see Figure 2).

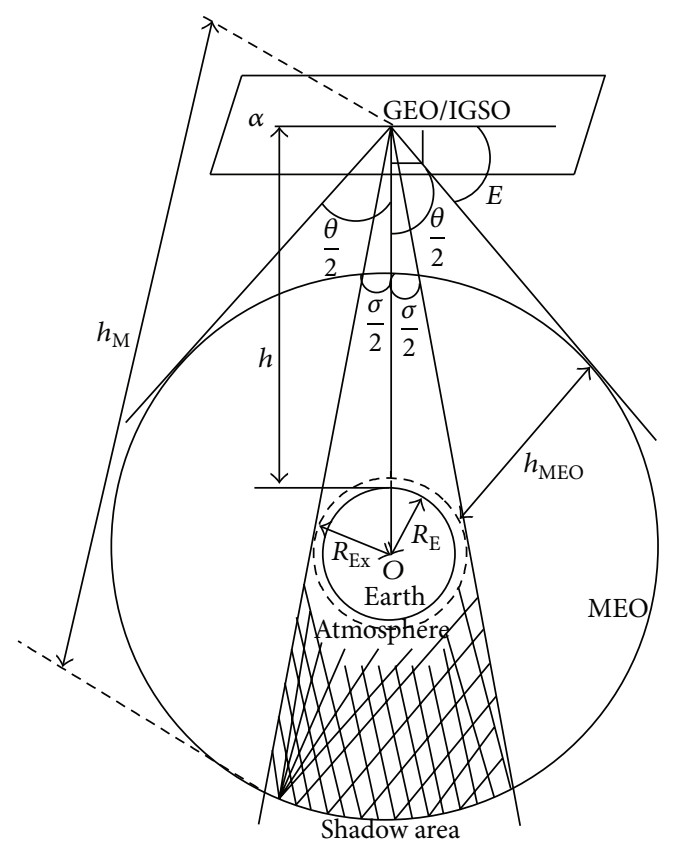

FIgURE 2: The visibility chart of STT\&C to MEO satellite.

In Figure 2, some relative quantities about the pitch angle $E$ are given as follows: the plane $\alpha$ is a vertical plane about the line, which passes through GEO/IGSO satellite and the center of the Earth; $h$ is the altitude between GEO/IGSO satellite and the surface of the Earth; $\theta$ is the antenna beam angle of GEO/IGSO satellite to MEO satellite; $R_{\mathrm{E}}$ is the Earth radius; $h_{\mathrm{MEO}}$ is the distance between MEO satellite and apogee point; the pitch angle $E$ is also the line angle between the plane $\alpha$ and the line passing through GEO/IGSO satellite and MEO satellite. The pitch angle $E$ can be described as

$$
|E|=\frac{\pi}{2}-\frac{\theta}{2}=\frac{\pi}{2}-\arcsin \frac{R_{\mathrm{E}}+h_{\mathrm{MEO}}}{R_{\mathrm{E}}+h} .
$$

3.2. The Sufficient and Necessary Conditions about the Visibility and the Coverage Rate Calculation Model. From Figure 2, we can infer that the coverage range projection of GEO/IGSO satellite to MEO satellite on the Earth is a circle, which is the GEO/IGSO satellite projection center on the Earth. And the radius of the circle can be described as $\left(R_{\mathrm{E}}+h_{\mathrm{MEO}}\right) \sin B_{S}$. Here $B_{S}$ is the latitude of the circle center. It is one of the coverage conditions that the track of subsatellite point of MEO satellite is within this circle.

The sufficient and necessary visibility conditions of STT\&C to BDS MEO satellite must be met with these two conditions as follows:

(a) The pitch angle $E$ must be met:

$$
|E|>90^{\circ}-\frac{\theta_{r}}{2}
$$

where $\theta_{r}$ is the valid beam angle and $\theta_{r}<\theta$.

(b) GEO/IGSO satellite and MEO satellite can not be shielded by the Earth. In order to avoid the effect of 
the atmosphere to the received signals, we added $1000 \mathrm{Km}$ to the Earth radius. Thus in the shadow area of Figure 2, STT\&C and BDS MEO satellite can not see each other.

According to the two sufficient and necessary visibility conditions above, there are two visibility areas of STT\&C to BDS MEO satellite. The sufficient and necessary conditions of the first visibility area are

$$
\begin{aligned}
\frac{\pi}{2}-\frac{\delta}{2} & \geq|E|>\frac{\pi}{2}-\frac{\theta_{r}}{2}, \\
h_{M} & >R \geq\left[\left(R_{\mathrm{E}}+h\right)^{2}-R_{\mathrm{Ex}}^{2}\right]^{1 / 2},
\end{aligned}
$$

where $\delta / 2=\arcsin \left(R_{\mathrm{Ex}} /\left(R_{\mathrm{E}}+h\right)\right) ; R_{\mathrm{Ex}}=1000 \mathrm{Km} ; R$ is the distance between GEO/IGSO satellite and MEO satellite;

$$
\begin{aligned}
h_{M} & =\left(h+R_{\mathrm{E}}\right) \cos \left(\frac{\delta}{2}\right) \\
& +\sqrt{\left(h+R_{\mathrm{E}}\right)^{2} \cos ^{2}\left(\frac{\delta}{2}\right)-\left(h+R_{\mathrm{E}}\right)^{2}+\left(h_{\mathrm{MEO}}+R_{\mathrm{E}}\right)^{2}} .
\end{aligned}
$$

The sufficient and necessary conditions of the second visibility area are

$$
\begin{aligned}
& \frac{\pi}{2} \geq|E|>\frac{\pi}{2}-\frac{\sigma}{2}, \\
& {\left[\left(R_{\mathrm{E}}+h\right)^{2}-R_{\mathrm{Ex}}^{2}\right]^{1 / 2}>R \geq\left(h-h_{\mathrm{MEO}}\right) . }
\end{aligned}
$$

On the basis of sufficient and necessary conditions, the total time $\Sigma t_{i}$ tracked by STT\&C will be calculated when the MEO satellite is flying. Let the total flying time of the MEO satellite be $T$; the coverage rate calculation model can be described as

$$
\zeta=\frac{\Sigma t_{i}}{T}
$$

\section{The Precise Orbit Determination Model of BDS MEO Satellites Based on the Multifrequency Combination}

As measured using periodic event, in most cases will appear ambiguity problem. Moreover, for the wavelength of carrier signal is generally just about $20 \mathrm{~cm}$, it is really difficult to determine the phase ambiguity about the carrier signal, from tens of thousands of kilometers. The longer the carrier wavelength is, the easier it is to calculate its phase ambiguity. Here take BDS signals as an example; their service carrier frequencies broadcasted are as follows: B1 1561.098 Mhz, B2 1207.14 Mhz, and B3 1268.52 Mhz [14]. Currently BDS clock precision can be superior to $6 \mathrm{~ns}$ [3]. Moreover, Yang et al. achieved $6 \mathrm{~h}$ prediction accuracy better than $2 \mathrm{~ns}$ by using the AR model [15]. As we know, measured error due to the multipath of carrier phase observation is less than a quarter of the wavelength. Additionally referring to the literature [4], let us suppose the GEO/IGSO orbit consistency is on the $2 \mathrm{dm}$ level. Each carrier signal wavelength and its measurement precision of BDS are listed in Table 1.
TABLE 1: BDS wavelength and the observation precision of each carrier signal.

\begin{tabular}{lccc}
\hline Carrier signal & B1 & B2 & B3 \\
\hline Wavelength & $19.2 \mathrm{~cm}$ & $24.8 \mathrm{~cm}$ & $23.6 \mathrm{~cm}$ \\
\hline Phase noise & $0.192 \mathrm{~cm}$ & $0.248 \mathrm{~cm}$ & $0.236 \mathrm{~cm}$ \\
\hline Multipath error & $4.8 \mathrm{~cm}$ & $6.2 \mathrm{~cm}$ & $5.9 \mathrm{~cm}$ \\
\hline GEO/IGSO orbit error & & $2 \mathrm{dm}$ & \\
\hline GEO/IGSO clock error & & $2 \mathrm{~ns}$ & \\
\hline
\end{tabular}

4.1. The Triple-Frequency Combination Model of BDS. When the receivers, which are installed on BDS MEO satellites, receive signal from GEO/IGSO satellites, the measurement errors do not contain ionosphere and troposphere propagation delay errors because the area of signal propagation path is located in more than 20,000 kilometers above ground, especially due to sufficient and necessary conditions of (3) and (5). In the moment of $t$ epoch, considering the impact of satellite orbital error, satellite clock error, multipath error, and phase measurement noise, carrier phase observation equations of BDS B1, B2, and B3 signals can be written as

$$
\begin{aligned}
\bar{\rho}_{k, \mathrm{~B} 1}^{S}(t)= & \rho_{k}^{S}(t)-\lambda_{\mathrm{B} 1} \cdot N_{k, \mathrm{~B} 1}^{S}\left(t_{0}\right)+d_{\mathrm{orb}}^{S}(t)+c \\
& \cdot \delta t_{k}(t)-c \cdot \delta t^{S}(t)+m_{k, L-\mathrm{B} 1}^{S}(t) \\
& +\varepsilon_{k, L-\mathrm{B} 1}^{S}(t), \\
\bar{\rho}_{k, \mathrm{~B} 2}^{S}(t)= & \rho_{k}^{S}(t)-\lambda_{\mathrm{B} 2} \cdot N_{k, \mathrm{~B} 2}^{S}\left(t_{0}\right)+d_{\mathrm{orb}}^{S}(t)+c \\
& \cdot \delta t_{k}(t)-c \cdot \delta t^{S}(t)+m_{k, L-\mathrm{B} 2}^{S}(t) \\
& +\varepsilon_{k, L-\mathrm{B} 2}^{S}(t), \\
\bar{\rho}_{k, \mathrm{~B} 3}^{S}(t)= & \rho_{k}^{S}(t)-\lambda_{\mathrm{B} 3} \cdot N_{k, \mathrm{~B} 3}^{S}\left(t_{0}\right)+d_{\text {orb }}^{S}(t)+c \\
& \cdot \delta t_{k}(t)-c \cdot \delta t^{S}(t)+m_{k, L-\mathrm{B} 3}^{S}(t) \\
& +\varepsilon_{k, L-\mathrm{B} 3}^{S}(t),
\end{aligned}
$$

where $\bar{\rho}_{k, \mathrm{~B} 1}^{S}(t), \bar{\rho}_{k, \mathrm{~B} 2}^{S}(t)$, and $\bar{\rho}_{k, \mathrm{~B} 3}^{S}(t)$ are the phase measurements in meters in the moment of $t$ epoch; $\rho_{k}^{S}(t)$ is the ideal geometrical distance between the receiver of number $k$ MEO satellite and number $S$ GEO/IGSO satellite in meters in the moment of $t$ epoch; $\lambda_{\mathrm{B} 1}, \lambda_{\mathrm{B} 2}$, and $\lambda_{\mathrm{B} 3}$ separately stand for BDS B1, B2, and B3 carrier signal wavelength; $N_{k, \mathrm{~B} 1}^{S}\left(t_{0}\right), N_{k, \mathrm{~B} 2}^{S}\left(t_{0}\right)$, and $N_{k, \mathrm{~B} 3}^{S}\left(t_{0}\right)$, respectively, stand for the corresponding integer ambiguity of BDS B1, B2, and B3 carrier phase measurements; $d_{\text {orb }}^{S}(t)$ is the relative orbit equivalent distance error between number $k \mathrm{MEO}$ satellite and number $S$ GEO/IGSO satellite; $\delta t_{k}(t), \delta t^{S}(t)$ separately stand for the corresponding receiver clock biases and satellite clock biases; $m_{k, L-\mathrm{B} 1}^{S}(t), m_{k, L-\mathrm{B} 2}^{S}(t)$, and $m_{k, L-\mathrm{B} 3}^{S}(t)$, respectively, stand for the multipath error; $\varepsilon_{k, L-\mathrm{B} 1}^{S}(t), \varepsilon_{k, L-\mathrm{B} 2}^{S}(t)$, and $\varepsilon_{k, L-\mathrm{B} 3}^{S}(t)$, respectively, stand for each phase measurement noise which corresponded to carrier measurements. 
Referring to Cocard et al. [16], the triple-frequency combination observation equation of BDS can be given as follows:

$$
\Phi_{k, \mathrm{TC}}^{S}(t)=\alpha \cdot \bar{\rho}_{k, \mathrm{~B} 1}^{S}(t)+\beta \cdot \bar{\rho}_{k, \mathrm{~B} 2}^{S}(t)+\gamma \cdot \bar{\rho}_{k, \mathrm{~B} 3}^{S}(t),
$$

where $\Phi_{k \text {,TC }}^{S}(t)$ is the virtual phase measurement of triplefrequency combination in the moment of $t$ epoch; subscript TC stands for the abbreviation of triple-frequency combination; $\alpha, \beta$, and $\gamma$ are combination coefficient.

By substituting (7) in (8), the expression for the triple-frequency combination observation equation of BDS becomes

$$
\begin{aligned}
& \Phi_{k, \mathrm{TC}}^{S}(t)=\rho_{k}^{S}(t) \cdot(\alpha+\beta+\gamma)-\left[\alpha \cdot \lambda_{\mathrm{B} 1} \cdot N_{k, \mathrm{~B} 1}^{S}\left(t_{0}\right)\right. \\
& \left.+\beta \cdot \lambda_{\mathrm{B} 2} \cdot N_{k, \mathrm{~B} 2}^{S}\left(t_{0}\right)+\gamma \cdot \lambda_{\mathrm{B} 3} \cdot N_{k, \mathrm{~B} 3}^{S}\left(t_{0}\right)\right] \\
& +d_{\mathrm{orb}}^{S}(t) \cdot(\alpha+\beta+\gamma)+c \cdot \delta t_{k}(t) \cdot(\alpha+\beta+\gamma)-c \\
& \cdot \delta t^{S}(t) \cdot(\alpha+\beta+\gamma)+\left[\alpha \cdot m_{k, L-\mathrm{B} 1}^{S}(t)+\beta\right. \\
& \left.\cdot m_{k, L-\mathrm{B} 2}^{S}(t)+\gamma \cdot m_{k, L-\mathrm{B} 3}^{S}(t)\right]+\left[\alpha \cdot \varepsilon_{k, L-\mathrm{B} 1}^{S}(t)+\beta\right. \\
& \left.\cdot \varepsilon_{k, L-\mathrm{B} 2}^{S}(t)+\gamma \cdot \varepsilon_{k, L-\mathrm{B} 3}^{S}(t)\right],
\end{aligned}
$$

where $\rho_{k}^{S}(t) \cdot(\alpha+\beta+\gamma)$ is the virtual geometrical distance between the receiver of number $k$ MEO satellite and number $S$ GEO/IGSO satellite in the moment of $t$ epoch. Of course, when $\alpha+\beta+\gamma=1$, it is the real geometrical distance between the receiver of number $k$ MEO satellite and number $S$ GEO/IGSO satellite.

Let $\lambda_{\mathrm{TC}} \cdot N_{k, \mathrm{TC}}^{S}\left(t_{0}\right)=\alpha \cdot \lambda_{\mathrm{B} 1} \cdot N_{k, \mathrm{~B} 1}^{S}\left(t_{0}\right)+\beta \cdot \lambda_{\mathrm{B} 2}$. $N_{k, \mathrm{~B} 2}^{S}\left(t_{0}\right)+\gamma \cdot \lambda_{\mathrm{B} 3} \cdot N_{k, \mathrm{~B} 3}^{S}\left(t_{0}\right)$, where $\lambda_{\mathrm{TC}}$ stands for the virtual wavelength of the triple-frequency combination carrier phase measurement. Hence, the corresponding integer ambiguity $N_{k, \mathrm{TC}}^{S}\left(t_{0}\right)$ can be expressed by

$$
\begin{aligned}
N_{k, \mathrm{TC}}^{S}\left(t_{0}\right)= & \alpha \cdot \frac{\lambda_{\mathrm{B} 1}}{\lambda_{\mathrm{TC}}} \cdot N_{k, \mathrm{~B} 1}^{S}\left(t_{0}\right)+\beta \cdot \frac{\lambda_{\mathrm{B} 2}}{\lambda_{\mathrm{TC}}} \cdot N_{k, \mathrm{~B} 2}^{S}\left(t_{0}\right) \\
& +\gamma \cdot \frac{\lambda_{\mathrm{B} 3}}{\lambda_{\mathrm{TC}}} \cdot N_{k, \mathrm{~B} 3}^{S}\left(t_{0}\right) .
\end{aligned}
$$

Let

$$
\begin{aligned}
& l=\alpha \cdot \frac{\lambda_{\mathrm{B} 1}}{\lambda_{\mathrm{TC}}}, \\
& m=\beta \cdot \frac{\lambda_{\mathrm{B} 2}}{\lambda_{\mathrm{TC}}}, \\
& n=\gamma \cdot \frac{\lambda_{\mathrm{B} 3}}{\lambda_{\mathrm{TC}}} .
\end{aligned}
$$

By substituting (11) in (10), one obtains an explicit type:

$$
\begin{aligned}
N_{k, \mathrm{TC}}^{S}\left(t_{0}\right)=l & \cdot N_{k, \mathrm{~B} 1}^{S}\left(t_{0}\right)+m \cdot N_{k, \mathrm{~B} 2}^{S}\left(t_{0}\right)+n \\
& \cdot N_{k, \mathrm{~B} 3}^{S}\left(t_{0}\right)
\end{aligned}
$$

where $l, m$, and $n$ are triple-frequency combination coefficient; they are arbitrary integers in order to ensure cycle properties of $N_{k, \mathrm{TC}}^{S}\left(t_{0}\right)$.

Additionally, (11) can also be described as

$$
\begin{aligned}
& \alpha=l \cdot \frac{\lambda_{\mathrm{TC}}}{\lambda_{\mathrm{B} 1}}, \\
& \beta=m \cdot \frac{\lambda_{\mathrm{TC}}}{\lambda_{\mathrm{B} 2}}, \\
& \gamma=n \cdot \frac{\lambda_{\mathrm{TC}}}{\lambda_{\mathrm{B} 3}} .
\end{aligned}
$$

When $\alpha+\beta+\gamma=1$, the wavelength $\lambda_{\mathrm{TC}}$ of combination observation is

$$
\lambda_{\mathrm{TC}}=\frac{\lambda_{\mathrm{B} 1} \cdot \lambda_{\mathrm{B} 2} \cdot \lambda_{\mathrm{B} 3}}{\alpha \cdot \lambda_{\mathrm{B} 2} \cdot \lambda_{\mathrm{B} 3}+\beta \cdot \lambda_{\mathrm{B} 1} \cdot \lambda_{\mathrm{B} 3}+\gamma \cdot \lambda_{\mathrm{B} 1} \cdot \lambda_{\mathrm{B} 2}} .
$$

For $\lambda=c / f$, where $c$ is the speed of light in vacuum and $f$ is the frequency of carrier phase, hence, $\lambda_{\mathrm{TC}}$ can be given by

$$
\lambda_{\mathrm{TC}}=\frac{c}{\left(l \cdot f_{\mathrm{B} 1}+m \cdot f_{\mathrm{B} 2}+n \cdot f_{\mathrm{B} 3}\right)} \text {. }
$$

And the frequency $f_{\mathrm{TC}}$ of combination observation is given as

$$
f_{\mathrm{TC}}=l \cdot f_{\mathrm{B} 1}+m \cdot f_{\mathrm{B} 2}+n \cdot f_{\mathrm{B} 3} \text {. }
$$

When $\alpha+\beta+\gamma=1$, (9) can be simplified as

$$
\begin{aligned}
& \Phi_{k, \mathrm{TC}}^{S}(t)=\rho_{k}^{S}(t)-\lambda_{\mathrm{TC}} \cdot N_{k, \mathrm{TC}}^{S}\left(t_{0}\right)+d_{\mathrm{orb}}^{S}(t)+c \\
& \quad \cdot \delta t_{k}(t)-c \cdot \delta t^{S}(t) \\
& +\left[\alpha \cdot m_{k, L-\mathrm{B} 1}^{S}(t)+\beta \cdot m_{k, L-\mathrm{B} 2}^{S}(t)+\gamma \cdot m_{k, L-\mathrm{B} 3}^{S}(t)\right] \\
& \quad+\left[\alpha \cdot \varepsilon_{k, L-\mathrm{B} 1}^{S}(t)+\beta \cdot \varepsilon_{k, L-\mathrm{B} 2}^{S}(t)+\gamma \cdot \varepsilon_{k, L-\mathrm{B} 3}^{S}(t)\right] .
\end{aligned}
$$

4.2. The Precision Orbit Determination Model of TripleFrequency Combination of BDS. Carrier phase measurement noise error is usually one percent of its wavelength. According to error propagation law, carrier measurement noise $\delta_{\mathrm{TC}}$ of BDS triple-frequency combination can be expressed as $\delta_{\mathrm{TC}}=\sqrt{\alpha^{2} \cdot \delta_{\mathrm{B} 1}^{2}+\beta^{2} \cdot \delta_{\mathrm{B} 2}^{2}+\gamma^{2} \cdot \delta_{\mathrm{B} 3}^{2}}$. On the basis of triplefrequency combination model of BDS above, according to the selection criteria and constraint conditions of combination coefficients $[14,17]$, considering the properties of (7) and ensuring the magnitude of error $\delta_{\mathrm{TC}}$ is of $\mathrm{cm}$ level, the combination coefficients and their other properties of some wide-lane (WL) and extra-wide-lane (EWL) of BDS triplefrequency combination are listed in Table 2. 
TABLE 2: BDS multifrequency combination observation and their properties.

\begin{tabular}{|c|c|c|c|c|c|c|c|c|c|c|}
\hline \multirow{2}{*}{ Carrier signal } & \multirow{2}{*}{ Combination } & \multicolumn{6}{|c|}{ Combination coefficient } & \multirow{2}{*}{$\begin{array}{l}\text { Combination } \\
\text { frequency/MHz }\end{array}$} & \multirow{2}{*}{$\begin{array}{c}\text { Combination } \\
\text { wavelength/m }\end{array}$} & \multirow{2}{*}{$\begin{array}{l}\text { Combination } \\
\text { phase noise/m }\end{array}$} \\
\hline & & $l$ & $m$ & $n$ & $\alpha$ & $\beta$ & $\gamma$ & & & \\
\hline B2-B3 & EWL & 0 & -1 & 1 & 0 & -19.69 & 20.69 & 61.38 & 4.884 & 0.069 \\
\hline B1-B2-B3 & $\mathrm{WL}$ & 1 & 1 & -2 & 6.75 & 5.23 & -10.99 & 231.198 & 1.297 & 0.032 \\
\hline B1-B3 & WL & 1 & 0 & -1 & 5.34 & 0 & -4.34 & 292.578 & 1.025 & 0.014 \\
\hline B1-B2-B3 & WL & 1 & -2 & 1 & 3.76 & -5.82 & 3.06 & 415.338 & 0.722 & 0.018 \\
\hline B1-B2-B3 & WL & 1 & -3 & 2 & 3.28 & -7.62 & 5.32 & 476.718 & 0.629 & 0.023 \\
\hline $\mathrm{B} 1-\mathrm{B} 2-\mathrm{B} 3$ & $\mathrm{WL}$ & 2 & 1 & -3 & 5.97 & 2.31 & -7.28 & 523.776 & 0.573 & 0.021 \\
\hline
\end{tabular}

In order to realize precise orbit determination for BDS MEO satellites, if we choose the EWL of B2-B3 carrier combination in Table 2, (17) can be recovered as follows:

$$
\begin{aligned}
\Phi_{k, \mathrm{TC}}^{S}(t)= & \rho_{k}^{S}(t)-4.884 N_{k, \mathrm{TC}}^{S}\left(t_{0}\right)+d_{\mathrm{orb}}^{S}(t)+c \\
& \cdot \delta t_{k}(t)-c \cdot \delta t^{S}(t)-19.69 m_{k, L-\mathrm{B} 2}^{S}(t) \\
& +20.69 m_{k, L-\mathrm{B} 3}^{S}(t)-19.69 \varepsilon_{k, L-\mathrm{B} 2}^{S}(t) \\
& +20.69 \varepsilon_{k, L-\mathrm{B} 3}^{S}(t),
\end{aligned}
$$

where $\rho_{k}^{S}(t)=\left[\left(X^{S}(t)-x_{k}(t)\right)^{2}+\left(Y^{S}(t)-y_{k}(t)\right)^{2}+\left(Z^{S}(t)-\right.\right.$ $\left.\left.z_{k}(t)\right)^{2}\right]^{1 / 2} ;\left[\begin{array}{lll}X^{S}(t) & Y^{S}(t) & Z^{S}(t)\end{array}\right]^{T}$ is the space coordinate of number $S$ GEO/IGSO satellite in the measurement of $t$ epoch; $\left[\begin{array}{lll}x_{k}(t) & y_{k}(t) & z_{k}(t)\end{array}\right]^{T}$ represents the space coordinate of number $k$ MEO satellite in the measurement of $t$ epoch.

For $\rho_{k}^{S}(t)$ is the nonlinear function about $\left[\begin{array}{lll}x_{k}(t) & y_{k}(t) & z_{k}(t)\end{array}\right]^{T}$, which are space coordinates of number $k$ MEO satellite in the moment of $t$ epoch, it should be linearized when it is processed by computer.

Assuming that $\left[\begin{array}{lll}\left.x_{k}(t)\right|_{0} & \left.y_{k}(t)\right|_{0} & \left.z_{k}(t)\right|_{0}\end{array}\right]^{T}$ is the approximate coordinate of number $k$ MEO satellite in the moment of $t$ epoch, letting

$$
\begin{aligned}
& x_{k}(t)=\left.x_{k}(t)\right|_{0}+\delta x_{k}(t), \\
& y_{k}(t)=\left.y_{k}(t)\right|_{0}+\delta y_{k}(t), \\
& z_{k}(t)=\left.z_{k}(t)\right|_{0}+\delta z_{k}(t),
\end{aligned}
$$

we can deduce the linearization formula of (18) as follows:

$$
\begin{aligned}
\Phi_{k, \mathrm{TC}}^{S}(t)= & \left(\rho_{k}^{S}(t)\right)_{0}-a_{k}^{S}(t) \cdot \delta x_{k}(t)-b_{k}^{S}(t) \cdot \delta y_{k}(t) \\
& -c_{k}^{S}(t) \cdot \delta z_{k}(t)-4.884 N_{k, \mathrm{TC}}^{S}\left(t_{0}\right) \\
& +d_{\mathrm{orb}}^{S}(t)+c \cdot \delta t_{k}(t)-c \cdot \delta t^{S}(t) \\
& -19.69 m_{k, L-\mathrm{B} 2}^{S}(t)+20.69 m_{k, L-\mathrm{B} 3}^{S}(t) \\
& -19.69 \varepsilon_{k, L-\mathrm{B} 2}^{S}(t)+20.69 \varepsilon_{k, L-\mathrm{B} 3}^{S}(t),
\end{aligned}
$$

where $a_{k}^{S}(t), b_{k}^{S}(t), c_{k}^{S}(t)$ are the coefficients of the linearization formula and $\left[\begin{array}{lll}a_{k}^{S}(t) & b_{k}^{S}(t) & c_{k}^{S}(t)\end{array}\right]^{T}$ is also regarded as the direction cosines vector from number $k$ MEO satellite to number $S$ GEO/IGSO satellite in the moment of $t$ epoch.
Generally, when the receiver of number $k$ MEO satellite observes continuously for a time, the number $n_{\Sigma}$ of the carrier observation equations would be greater than the total number $n_{u}$ of the unknown parameters. When $n_{\Sigma}>n_{u}$, the error matrix equation with respect to the unknown parameters of (20) can be given as

$$
\underset{n_{\Sigma} \times 1}{\mathbf{V}_{k}(t)}=\underset{n_{\Sigma} \times n_{u}}{\mathbf{B}_{k}(t)} \delta \mathbf{G}_{k} \times 1=\underset{n_{\Sigma} \times 1}{\mathbf{L}_{k}(t)}
$$

where $\mathbf{B}_{k}(t)$ is the coefficient matrix with respect to the unknown parameters; $\delta \mathbf{G}_{k}(t)$ is the matrix with respect to the unknown parameters; $\mathbf{L}_{k}(t)$ is the matrix of constant term.

According to Gaussian least-square principle, in order to calculate the matrix $\delta \mathbf{G}_{k}(t)$ with respect to the unknown parameters in (21), the condition of $\mathbf{V}_{i}^{T}(t) \mathbf{V}_{i}(t)=$ min must be satisfied. Referring to the mathematical function extremum method, one leads to

$$
\frac{\partial \mathbf{V}_{k}^{T}(t) \mathbf{V}_{k}(t)}{\partial \delta \widehat{\mathbf{G}}_{k}(t)}=2 \mathbf{V}_{k}^{T}(t) \mathbf{B}_{k}(t)=0
$$

Transposition leads to

$$
\mathbf{B}_{k}^{T}(t) \mathbf{V}_{k}(t)=0
$$

By substituting (21) in (23), the optimal value $\delta \widehat{\mathbf{G}}_{k}(t)$ of the matrix $\delta \mathbf{G}_{k}(t)$ with respect to the unknown parameters can be calculated by

$$
\begin{aligned}
\delta \widehat{\mathbf{G}}_{k}(t) & =\left(\mathbf{B}_{k}^{T}(t) \mathbf{B}_{k}(t)\right)^{-1} \mathbf{B}_{k}^{T}(t) \mathbf{L}_{k}(t) \\
& =\widehat{\mathbf{Q}}_{\delta \widehat{\mathbf{G}} \delta \widehat{\mathbf{G}}} \mathbf{B}_{k}^{T}(t) \mathbf{L}_{k}(t),
\end{aligned}
$$

where $\widehat{\mathbf{Q}}_{\delta \widehat{\mathbf{G}} \delta \widehat{\mathbf{G}}}$ is the corresponding cofactor matrix about $\delta \widehat{\mathbf{G}}_{k}(t)$.

4.3. The Orbit Determination Accuracy Evaluation Model of $M E O$ Satellite. Referring to the error propagation law, the corresponding mean square error $\delta_{0}$ with respect to combination carrier measurement can be expressed as follows:

$$
\delta_{0}= \pm \sqrt{\frac{\mathbf{V}_{k}^{T}(t) \cdot \mathbf{V}_{k}(t)}{f}}
$$

where $f=n_{\Sigma}-n_{u}$. 

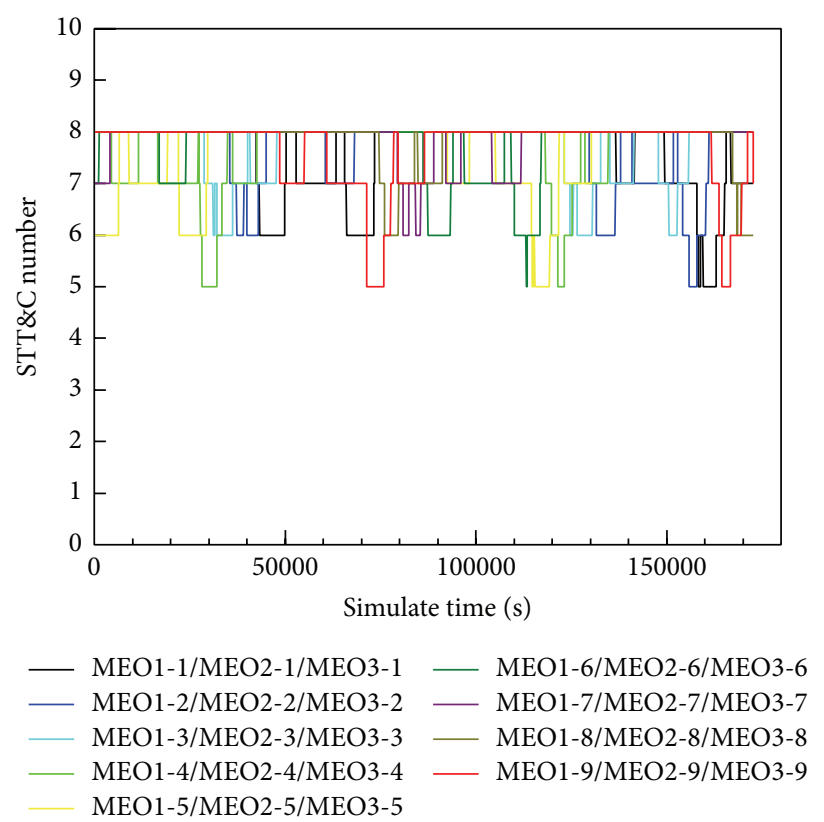

(a) MEO1 orbit

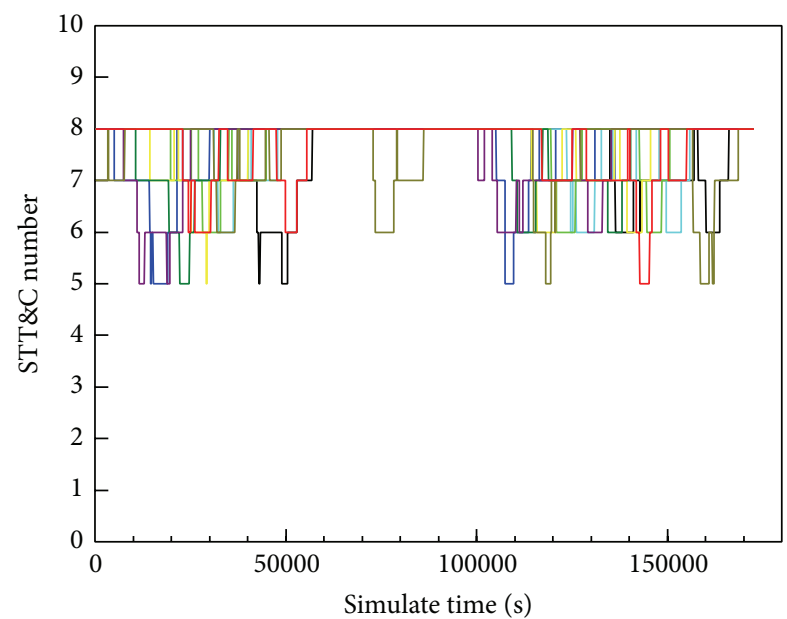

$\begin{array}{rr}\text { MEO1-1/MEO2-1/MEO3-1 } & \text { MEO1-6/MEO2-6/MEO3-6 } \\ \text { MEO1-2/MEO2-2/MEO3-2 } & \text { MEO1-7/MEO2-7/MEO3-7 } \\ \text { MEO1-3/MEO2-3/MEO3-3 } & \text { MEO1-8/MEO2-8/MEO3-8 } \\ \text { MEO1-4/MEO2-4/MEO3-4 } & \text { MEO1-9/MEO2-9/MEO3-9 } \\ \text { MEO1-5/MEO2-5/MEO3-5 } & \end{array}$

(b) MEO2 orbit
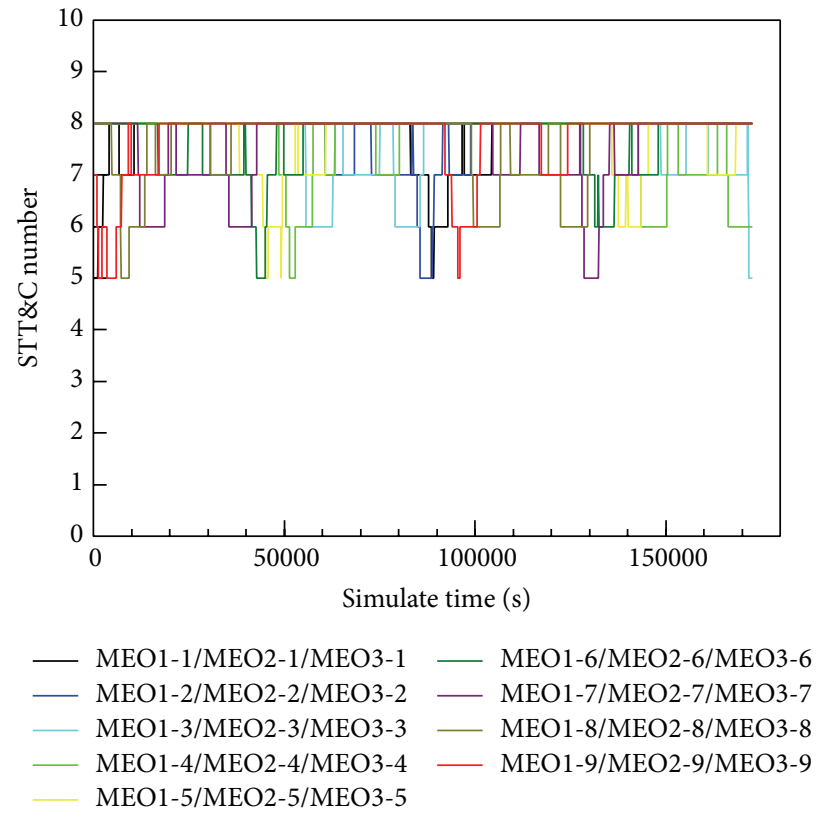

(c) MEO3 orbit

FIGURE 3: The visibility number of STT\&C to BDS MEO satellite.

Thus the accuracy evaluation equation of the optimal value $\delta \widehat{\mathbf{G}}_{k}(t)$ can be expressed as follows:

$$
\left(m_{\delta \widehat{\mathbf{G}}_{k}}\right)_{j}=\delta_{0} \sqrt{\left(\widehat{\mathbf{Q}}_{\delta \widehat{G}_{k} \delta \widehat{G}_{k}}\right)_{j j}}
$$

where $\left(\widehat{\mathbf{Q}}_{\delta \widehat{\mathbf{G}}_{k} \delta \widehat{\mathbf{G}}_{k}}\right)_{j j}$ is the main number $j$ diagonal element of $\widehat{\mathbf{Q}}_{\delta \widehat{\mathbf{G}} \delta \widehat{\mathbf{G}}}$.
The PDOP (Position Dilution of Precision) of the orbit determination of MEO satellite in the moment of $t$ epoch can be described as

$$
\mathrm{PDOP}=\sqrt{\mathrm{Q}_{\delta \bar{x}_{k}(t) \delta \bar{x}_{k}(t)}+Q_{\delta \hat{y}_{k}(t) \delta \hat{y}_{k}(t)}+Q_{\delta \bar{z}_{k}(t) \delta \bar{z}_{k}(t)}} .
$$

Additionally the corresponding horizontal precision of the orbit determination of MEO satellite in the moment of $t$ epoch can be also represented as

$$
m_{P}(t)=\delta_{0} \cdot \text { PDOP. }
$$



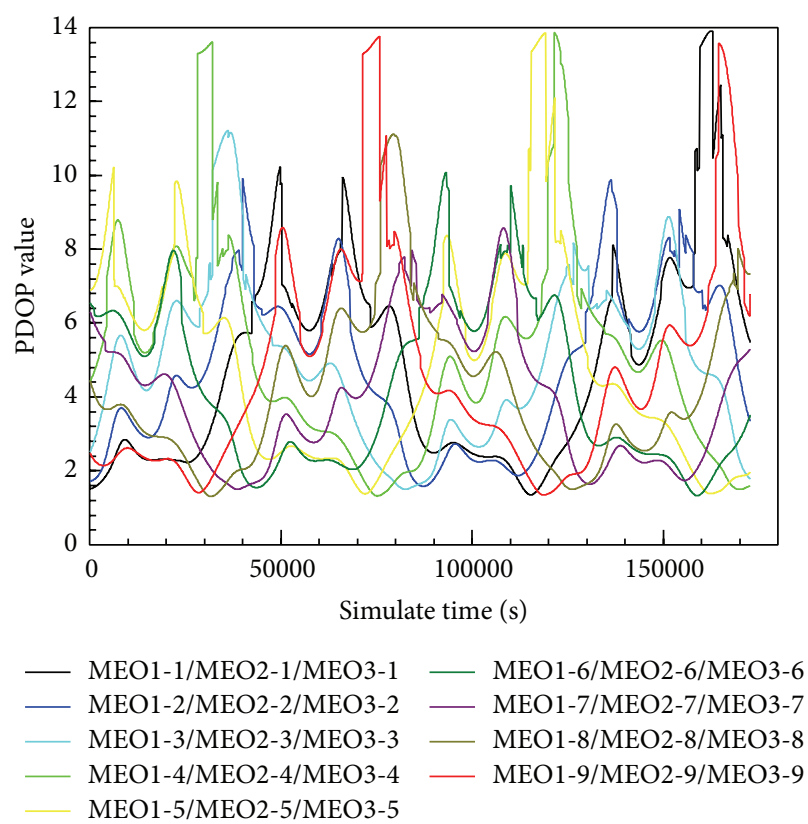

(a) MEO1 orbit
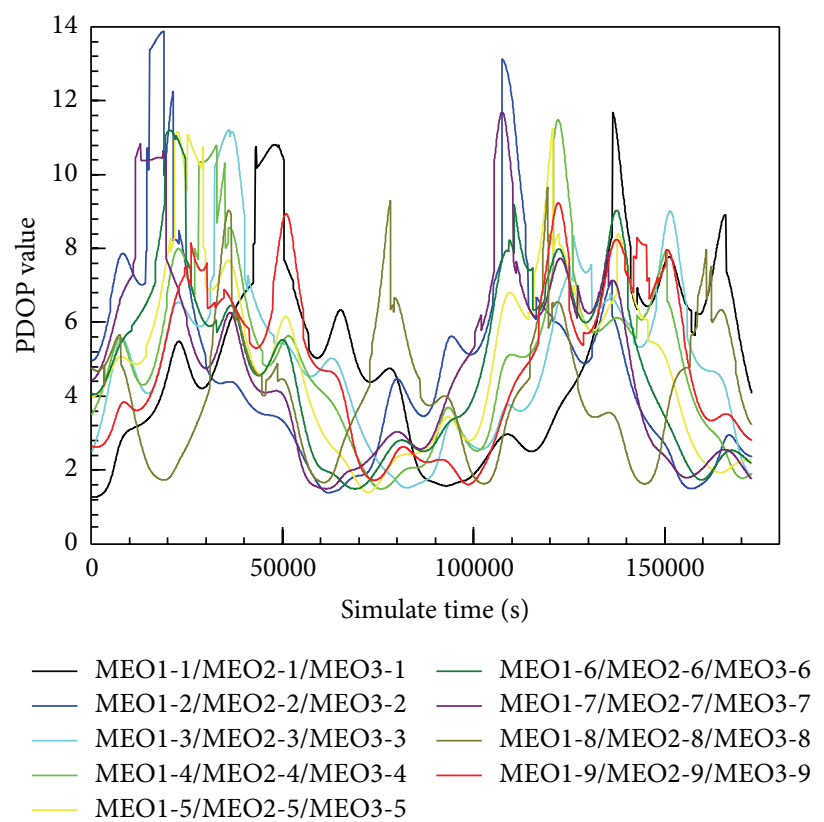

(b) $\mathrm{MEO} 2$ orbit
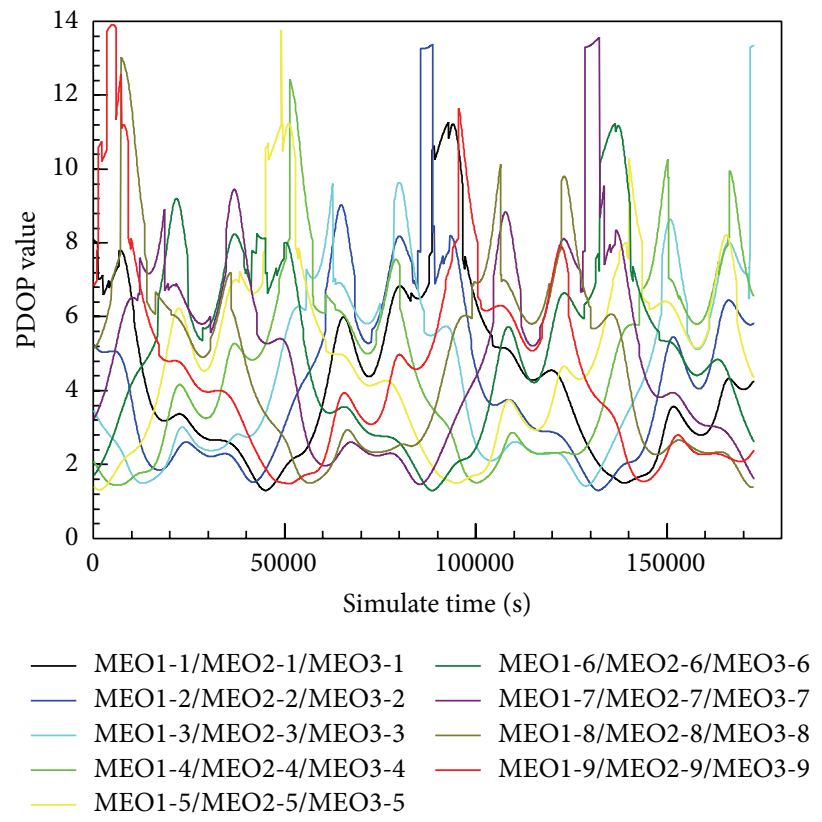

(c) $\mathrm{MEO} 3$ orbit

FIGURE 4: PDOP value of BDS MEO satellite.

The HDOP (Horizontal Dilution of Precision) of the orbit determination of MEO satellite in the moment of $t$ epoch can be described as

$$
\mathrm{HDOP}=\sqrt{\mathrm{Q}_{\delta \bar{x}_{k}(t) \delta \bar{x}_{k}(t)}+Q_{\delta \hat{y}_{k}(t) \delta \hat{y}_{k}(t)}} .
$$

The corresponding horizontal precision of the orbit determination of MEO satellite in the moment of $t$ epoch can be also represented as

$$
m_{H}(t)=\delta_{0} \cdot \text { HDOP. }
$$

The VDOP (Vertical Dilution of Precision) of the orbit determination of MEO satellite in the moment of $t$ epoch can be described as

$$
\mathrm{VDOP}=\sqrt{\mathrm{Q}_{\delta \bar{z}_{k}(t) \delta \bar{z}_{k}(t)}} .
$$

The corresponding vertical precision of the orbit determination of MEO satellite in the moment of $t$ epoch can be also represented as

$$
m_{V}(t)=\delta_{0} \cdot \text { VDOP. }
$$



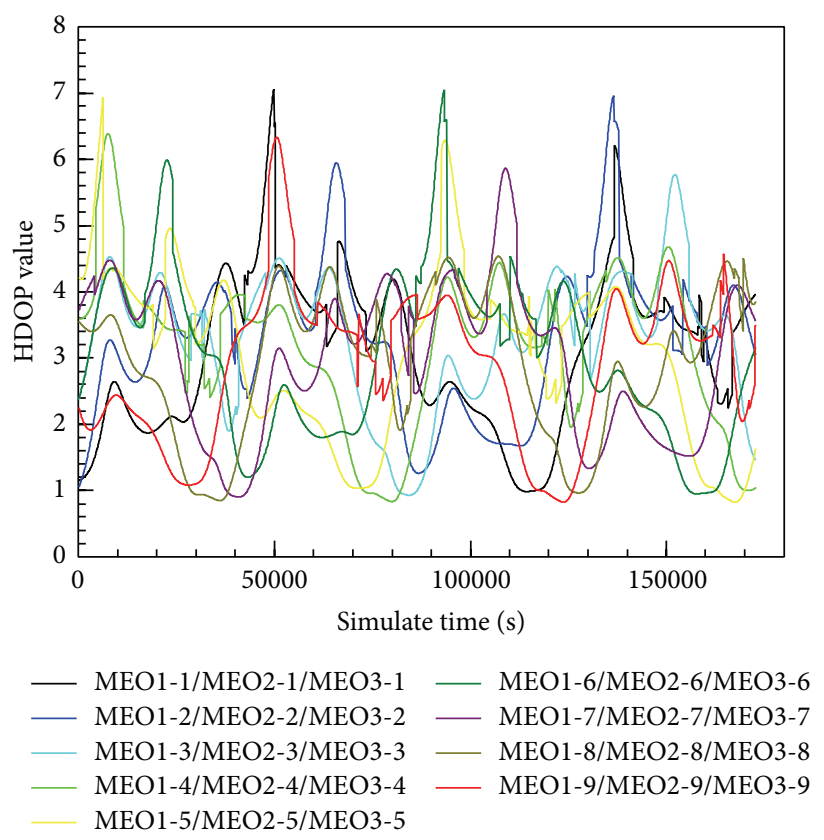

(a) MEO1 orbit
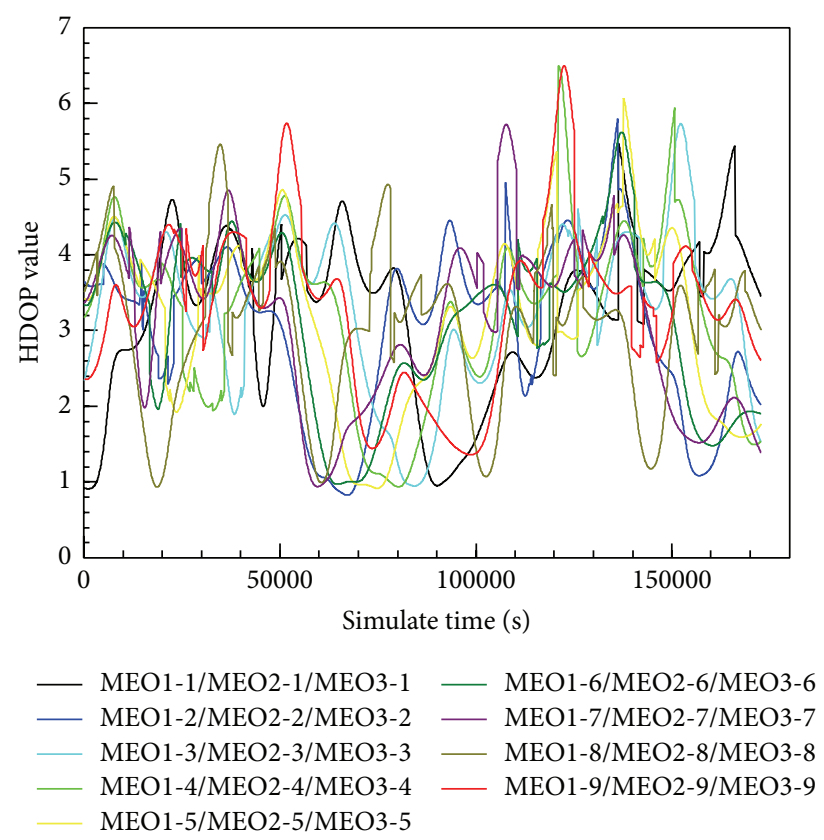

(b) $\mathrm{MEO} 2$ orbit
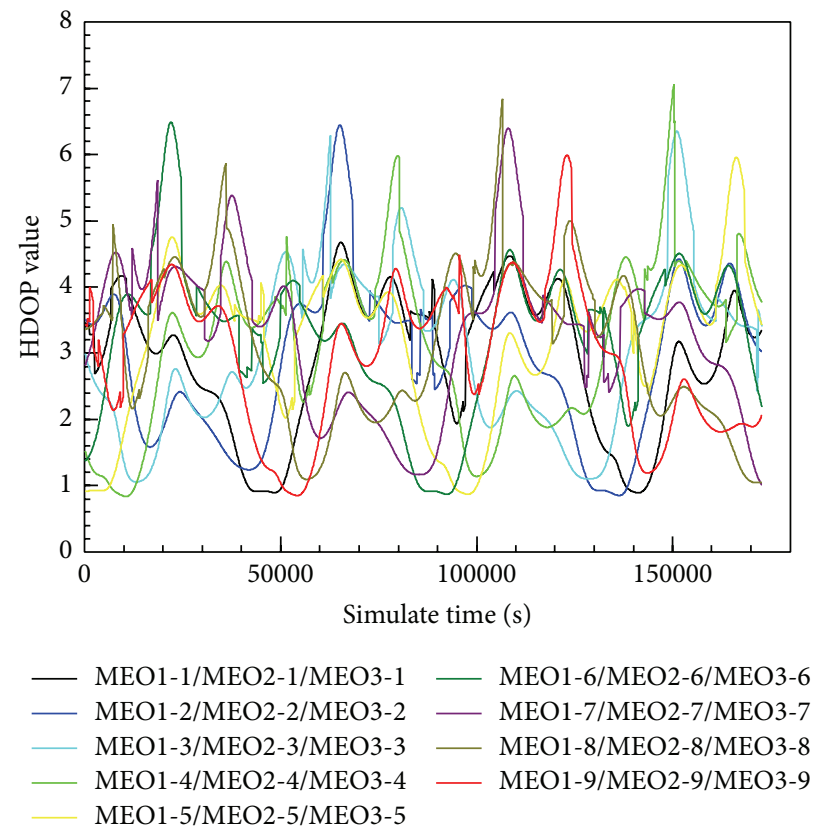

(c) $\mathrm{MEO} 3$ orbit

Figure 5: HDOP value of BDS MEO satellite.

\section{Performance Analysis of the Precise Orbit Determination of BDS MEO Satellite}

On the basis of the visibility and coverage rate calculation model above, the simulation calculation has been designed here. According to the literature [18], we select the simulation parameters of BDS as follows: the five GEO satellites' orbital altitudes are all $35786 \mathrm{~km}$; the 27 MEO satellites' orbital altitudes are all $21528 \mathrm{~km}$, and their orbital inclinations are all 55 degrees; the three IGSO satellites' orbital altitudes are all $35786 \mathrm{~km}$, and their orbital inclinations are all 55 degrees too. Referring to the literature [11], let the beam angles of GEO/IGSO satellites be 160 degrees. According to these main parameters, the simulation results of two days are given in Figures 3-9.

Figure 3 shows the visibility number of 27 BDS MEO satellite receivers to STT\&C stations in the simulation period. Here MEO1, MEO2, and MEO3 stand for the orbital plane number. MEO1-1, MEO1-2, MEO1-3, ..., and MEO3-9 are the serial numbers of $27 \mathrm{BDS} \mathrm{MEO}$ satellites. As we can 

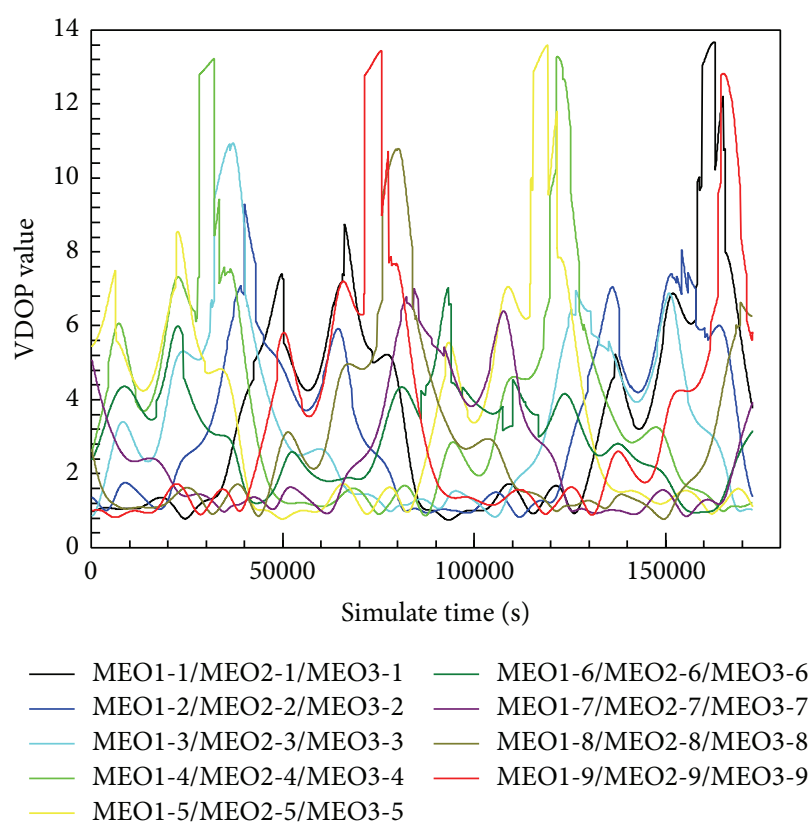

(a) MEO1 orbit
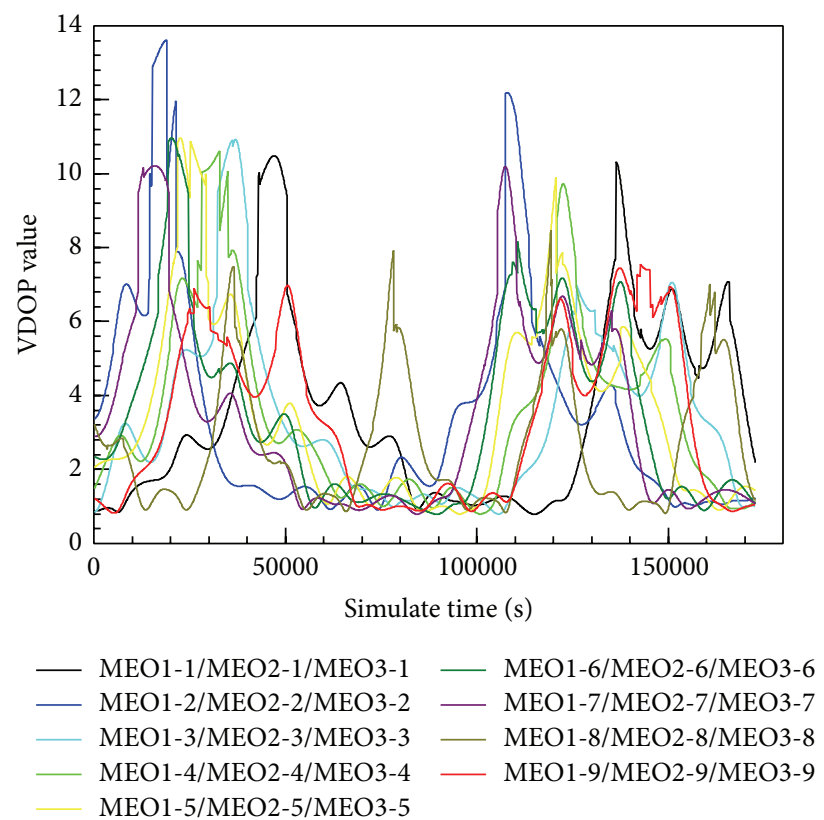

(b) $\mathrm{MEO} 2$ orbit
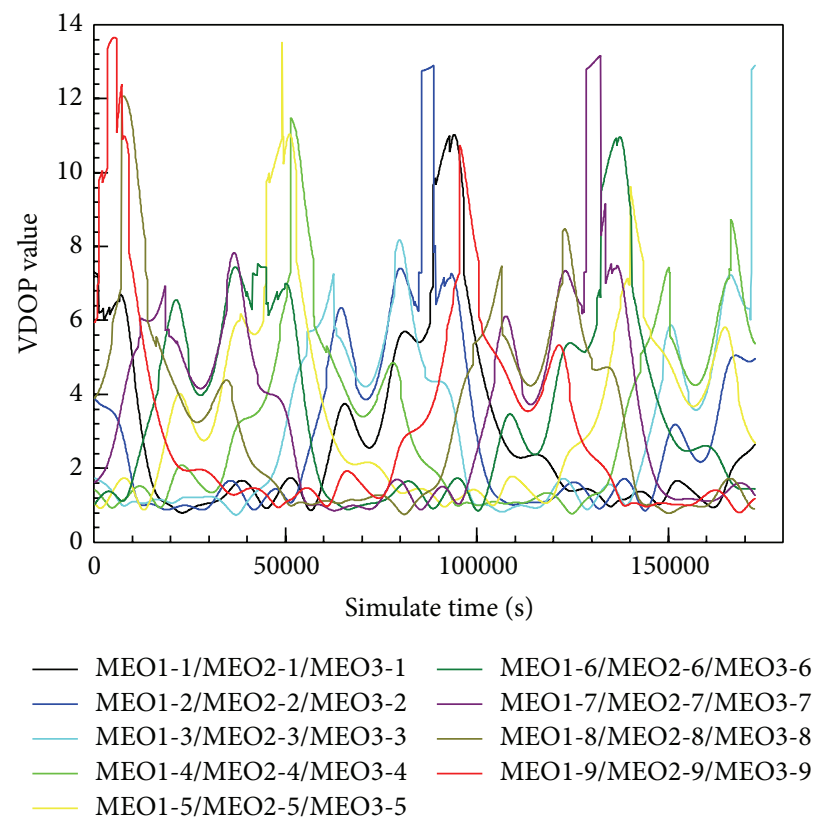

(c) $\mathrm{MEO} 3$ orbit

FIGURE 6: VDOP value of BDS MEO satellite.

see, most of the time, the visibility number is more than six and, for only a few times, the visibility number is five. Thus orbit determination of BDS MEO satellite based on STT\&C stations can be realized for all period time.

Figures 4-6 give the results of 27 BDS MEO satellite receivers' PDOP, HDOP, and VDOP with respect to STT\&C stations. It can be found from Figure 4, most of the time, that PDOP value is less than six, and about $50 \%$ of simulation period time, the value is less than 4 , while it is seldom greater than 10. From Figure 5, we can see, most of the time, that HDOP value is within 4 , while it is seldom slightly greater than 5. And Figure 6 shows that VDOP value is within 5 most of the period time, while it is seldom greater than 9 . Combining with Figure 3, we can see that the PDOP, HDOP, and VDOP value is at accord with the visibility number of 27 BDS MEO satellite receivers to STT\&C stations; that is, the greater the visibility number is, the smaller the value of PDOP, HDOP, and VDOP is.

Referring to the accuracy of Tables 1 and 2 given and according to the simulation design of the BDS constellation above, ephemeris data and observation data are simulated. Using the precise orbit determination model of BDS MEO 

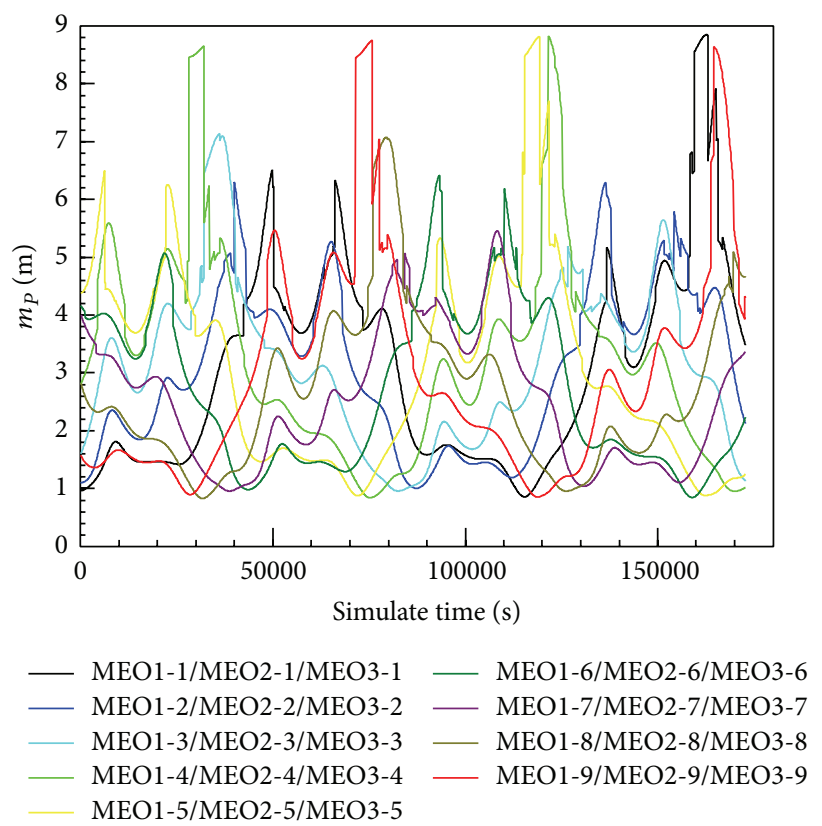

(a) MEO1 orbit
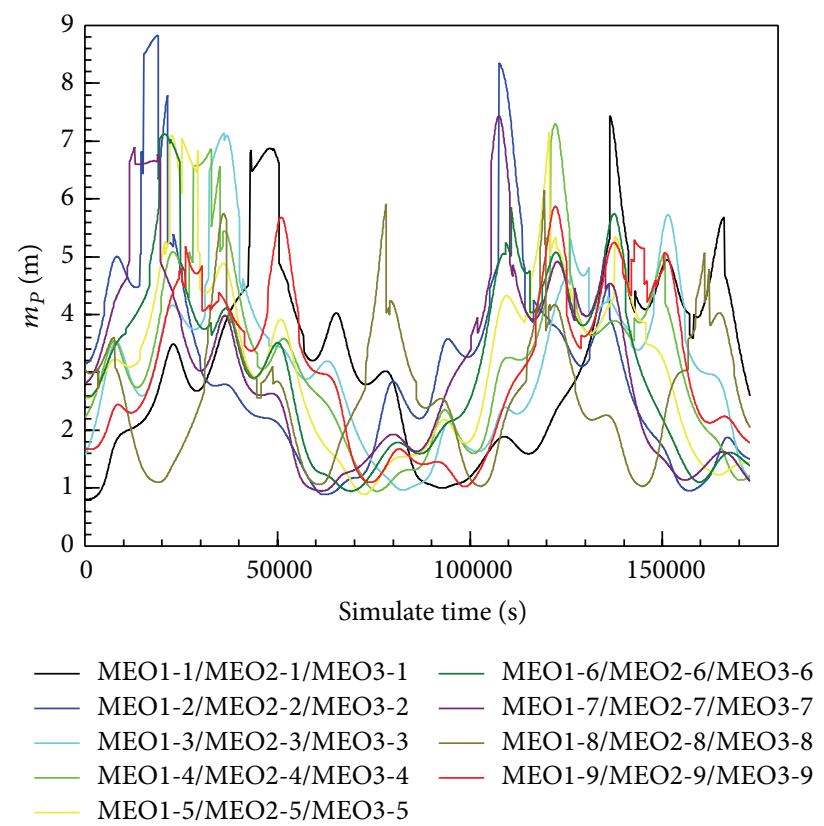

(b) $\mathrm{MEO} 2$ orbit
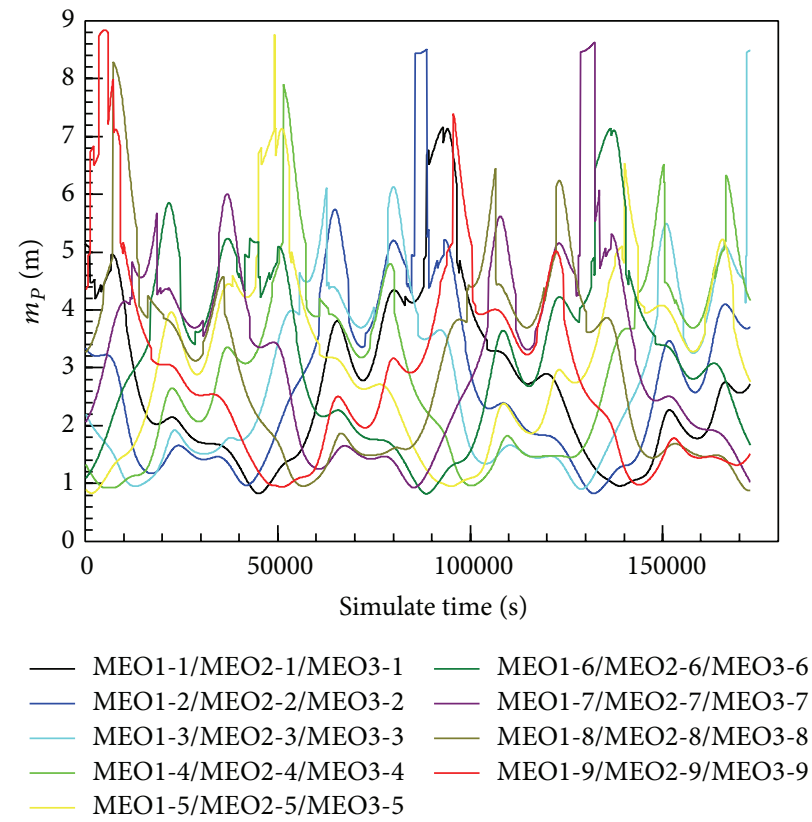

(c) $\mathrm{MEO} 3$ orbit

FIGURE 7: The simulation precision $m_{P}$ of the orbit determination of BDS MEO satellite.

satellite based on multifrequency combination above, the accuracy results of the orbit determination of BDS MEO satellites are showed in Figures 7-9.

Figures 7-9 give the results of $27 \mathrm{BDS}$ MEO satellite receivers' position accuracy $m_{P}$, horizontal accuracy $m_{H}$, and vertical accuracy $m_{V}$ based on STT\&C stations. It can be found from Figure 7, most of the time, that the position accuracy $m_{P}$ is less than $4 \mathrm{~m}$, and about $50 \%$ of simulation period time, the value is less than $3 \mathrm{~m}$, while it is seldom greater than $6 \mathrm{~m}$. From Figure 8, we can see, most of the time, that the horizontal accuracy $m_{H}$ is within $2.5 \mathrm{~m}$, and about $50 \%$ of simulation period time, the value is less than $2 \mathrm{~m}$, while it is seldom greater than $3 \mathrm{~m}$. And Figure 9 shows that the vertical accuracy $m_{V}$ is within $3.5 \mathrm{~m}$ most of the period time, and about $50 \%$ of simulation period time, the value is less than $3 \mathrm{~m}$, while it is seldom greater than $5 \mathrm{~m}$. Therefore it is one of the high and autonomous accuracy methods to determine BDS MEO satellite position by using STT\&C stations for all period time. 

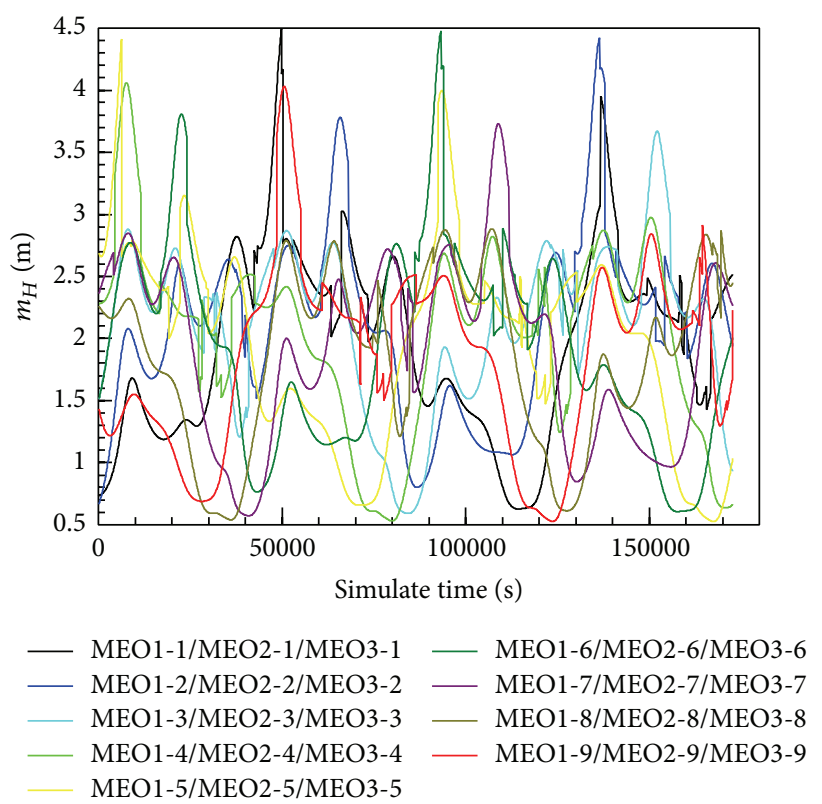

(a) MEO1 orbit
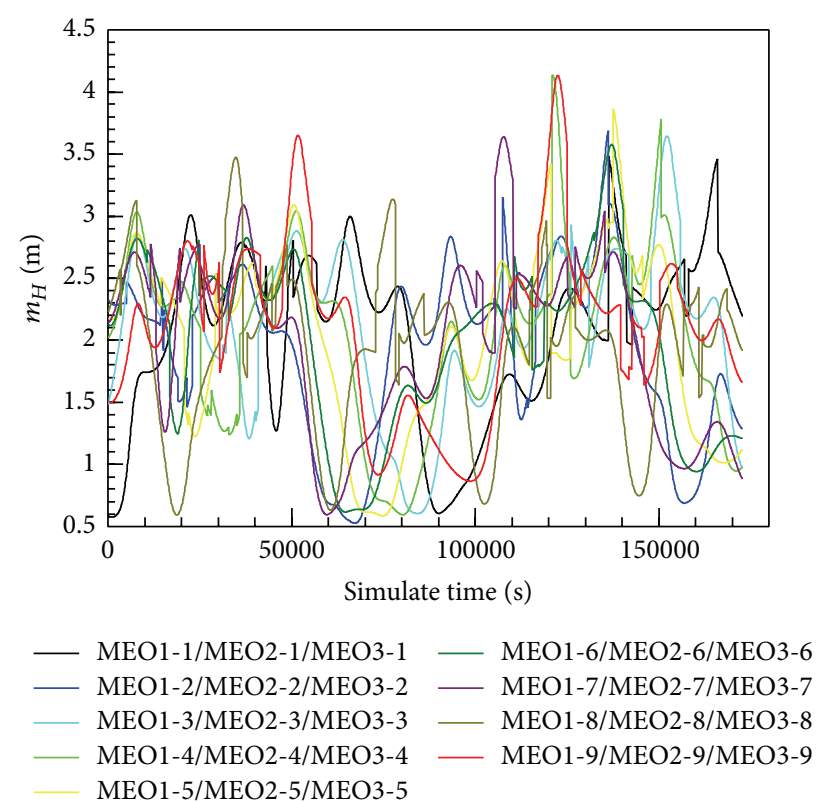

(b) $\mathrm{MEO} 2$ orbit

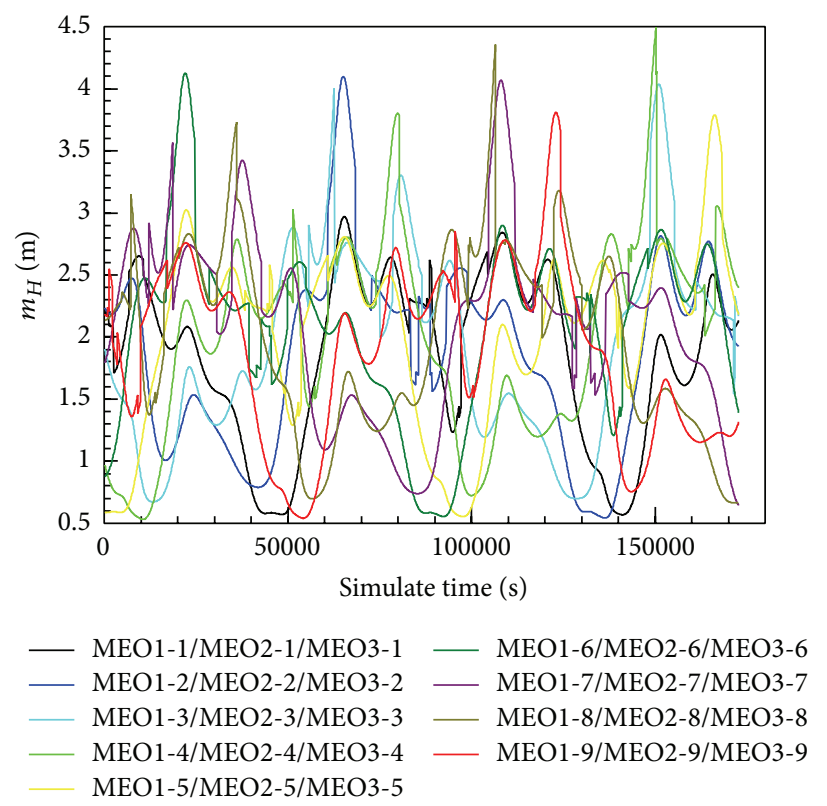

(c) $\mathrm{MEO} 3$ orbit

FIGURE 8: The simulation precision $m_{H}$ of the orbit determination of BDS MEO satellite.

\section{Conclusion}

It is difficult to realize the whole period and high precise orbit determination for BDS MEO satellites by using ground TT\&C stations or ground differential reference stations of the Chinese's own. The method of precise orbit determination of BDS MEO satellites based on STT\&C stations was proposed in this paper, the precise orbit determination model based on BDS EWL of B2-B3 combination was deduced, and its orbit determination accuracy was evaluated. The results show that the method and the precise orbit determination model of BDS MEO satellites based on the multifrequency combination mentioned in this paper can solve the problem of orbit determination with respect to BDS MEO satellites. Moreover, on the basis of the dm level orbit determination for GEO/IGSO satellites, this method can meet the meterlevel higher accuracy requirement and can realize precise orbit determination of BDS MEO satellites for all period time.

\section{Conflict of Interests}

The authors declare that there is no conflict of interests regarding the publication of this paper. 


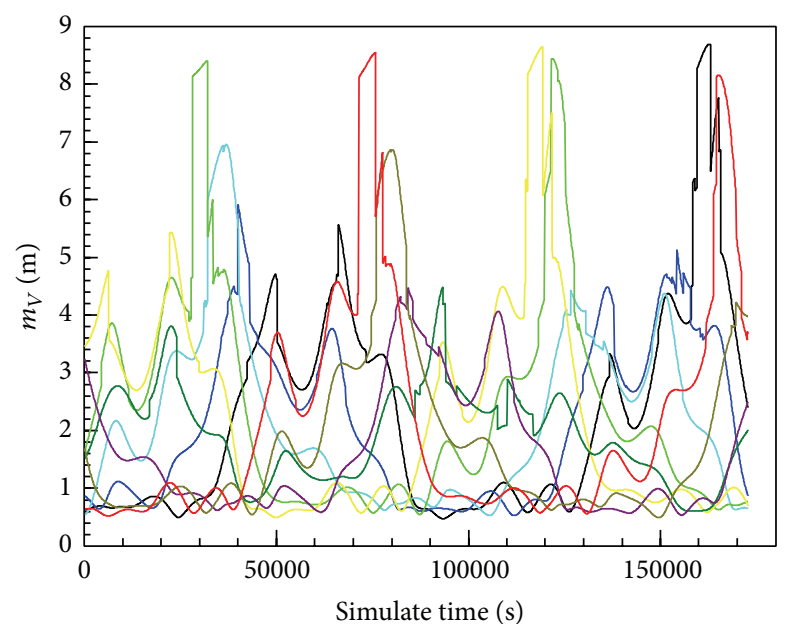

MEO1-1/MEO2-1/MEO3-1
MEO1-2/MEO2-2/MEO3-2 - MEO1-6/MEO2-6/MEO3-6
MEO1-3/MEO2-3/MEO3-3 - MEO1-7/MEO2-7/MEO3-7
MEO1-4/MEO2-4/MEO3-4 - MEO1-9/MEO2-9/MEO3-9
MEO1-5/MEO2-5/MEO3-5

(a) MEO1 orbit
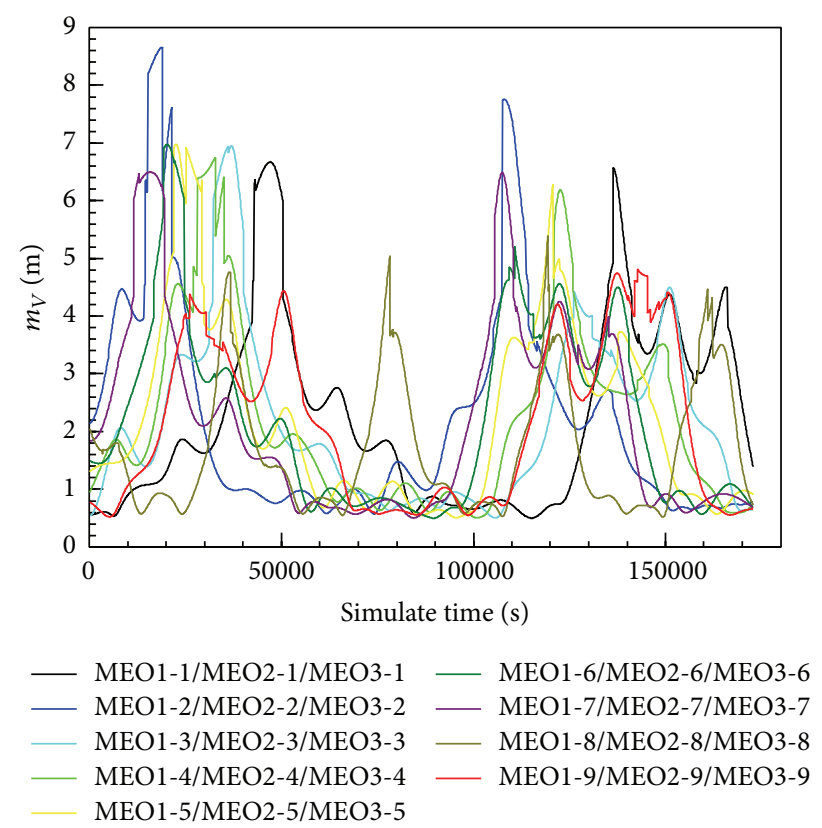

(b) $\mathrm{MEO} 2$ orbit
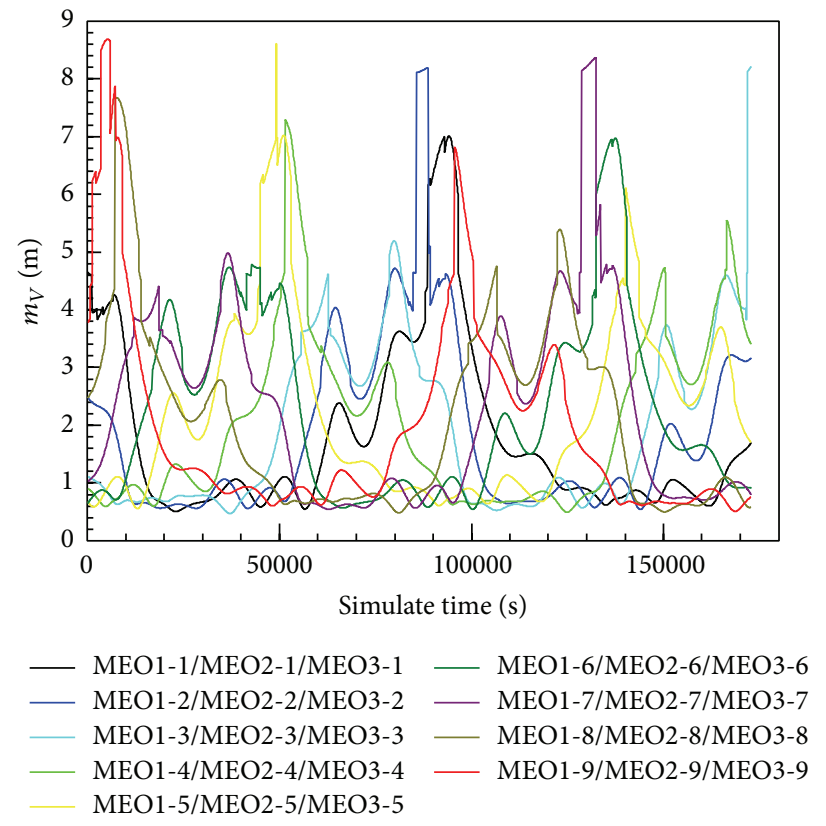

(c) $\mathrm{MEO} 3$ orbit

FIGURE 9: The simulation precision $m_{V}$ of the orbit determination of BDS MEO satellite.

\section{Acknowledgment}

This research was funded by the National Natural Science Foundation of China (nos. 41202245, 41272373).

\section{References}

[1] Y. Wen, Q. Liu, J. Zhu et al., "The effect of TT \& C deployment on the regional satellite navigation system," Journal of National University of Defense Technology, vol. 29, no. 1, pp. 1-6, 2007.
[2] K. Li, A. Ding, H. Zhang et al., "Augmentation of virtual satellite TT\&C station to BDS TT\&C network," Journal of Geomatics Science and Technology, vol. 31, no. 3, pp. 43-47, 2014.

[3] W. Gao, M. Su, S. Su et al., "Testing and evaluation of signal in space accuracy for Beidou Navigation Satellite System (BDS)," in Proceedings of the 4th China Satellite Navigation Conference, pp. 1-7, Wuhan, China, May 2013.

[4] P. Steigenberger, U. Hugentobler, A. Hauschild, and O. Montenbruck, "Orbit and clock analysis of Compass GEO and IGSO satellites," Journal of Geodesy, vol. 87, no. 6, pp. 515-525, 2013. 
[5] U. Vollath, S. Birnbach, H. Landau, J. M. Fraile-Ordoñez, and M. Martin-Neira, "Analysis of three-carrier ambiguity resolution (TCAR) technique for precise relative positioning in GNSS-2," in Proceedings of the 11th International Technical Meeting of the Satellite Division of the Institute of Navigation (ION GPS '98), pp. 417-426, Nashville,Tenn, USA, September 1998.

[6] P. J. de Jonge, P. J. G. Teunissen, N. F. Jonkman, and P. Joosten, "The distributional dependence of the range on triple frequency GPS ambiguity resolution," in Proceedings of the National Technical Meeting of the Institute of Navigation, pp. 605-612, Anaheim, Calif, USA, January 2000.

[7] R. Hatch, J. Jung, P. Enge, and B. Pervan, "Civilian GPS: the benefits of three frequencies," GPS Solutions, vol. 3, no. 4, pp. $1-9,2000$.

[8] D. Odijk and P. J. G. Teunissen, "ADOP in closed form for a hierarchy of multi-frequency single-baseline GNSS models," Journal of Geodesy, vol. 82, no. 8, pp. 473-492, 2008.

[9] Y. Feng and J. Wang, "Exploring GNSS RTK performance benefits with GPS and virtual Galileo measurements," in Proceedings of the 2007 National Technical Meeting of the Institute of Navigation (ION '07), pp. 218-226, San Diego, Calif, USA, January 2007.

[10] Y. Feng, "GNSS three carrier ambiguity resolution using ionosphere-reduced virtual signals," Journal of Geodesy, vol. 82, no. 12, pp. 847-862, 2008.

[11] J. Paziewski and P. Wielgosz, "Accounting for Galileo-GPS inter-system biases in precise satellite positioning," Journal of Geodesy, vol. 89, no. 1, pp. 81-93, 2015.

[12] B. Sun, J. Ou, X. Yang et al., "GEO navigation satellite precise orbit determination based on multi-frequency carrier phase combination," in Proceedings of the 1st China Satellite Navigation Conference, pp. 688-700, Beijing, China, May 2010.

[13] A. Renga, M. Grassi, and U. Tancredi, "Relative navigation in LEO by carrier-phase differential GPS with intersatellite ranging augmentation," International Journal of Aerospace Engineering, vol. 2013, Article ID 627509, 11 pages, 2013.

[14] J. Shen, H. He, H. Guo et al., "Application research of linear combination based on triple-frequency observation," GNSS World of China, vol. 37, no. 6, pp. 37-40, 2012.

[15] G. Yang, X. Xu, J. Cao, X. Liu, and Q. Wang, "Precision analysis for Compass satellite clock prediction based on a universal clock offset model," Scientia Sinica Physica, Mechanica \& Astronomica, vol. 45, no. 7, Article ID 079502, pp. 1-6, 2015.

[16] M. Cocard, S. Bourgon, O. Kamali, and P. Collins, "A systematic investigation of optimal carrier-phase combinations for modernized triple-frequency GPS," Journal of Geodesy, vol. 82, no. 9, pp. 555-564, 2008.

[17] H. Guo, H. He, J. Li et al., "Estimation and mitigation of the main erros for centimeter level compass RTK solutions over medium-long base lines," The Journal of Navigation, vol. 64, pp. 113-126, 2011.

[18] China Satellite Navigation Office, BeiDou Navigation Satellite System open service performance standard (Version 1.0), 2013. 


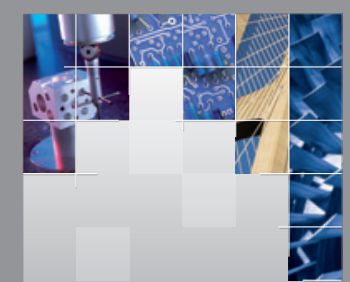

\section{Enfincering}
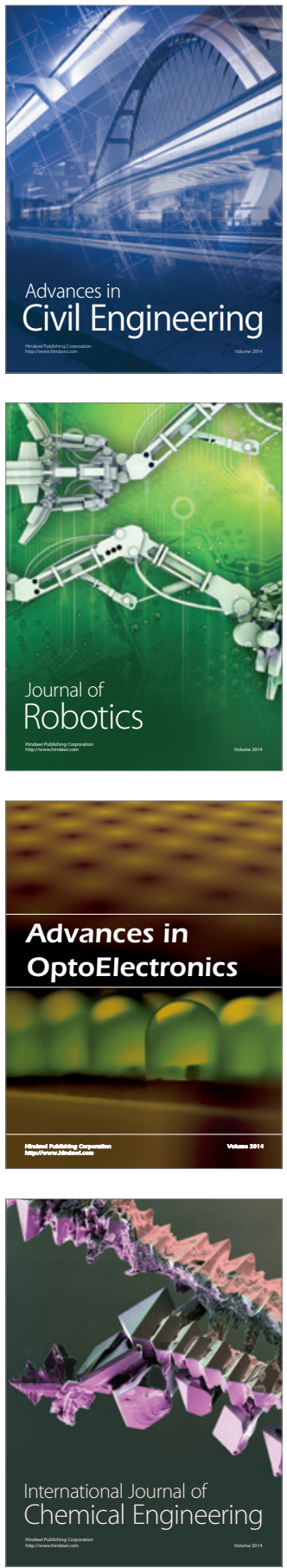

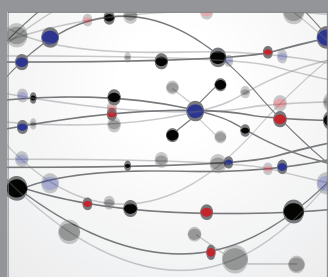

The Scientific World Journal

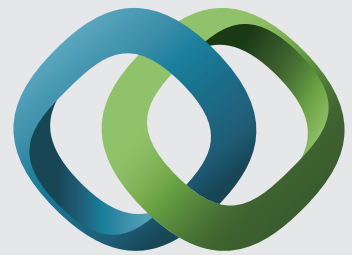

\section{Hindawi}

Submit your manuscripts at

http://www.hindawi.com
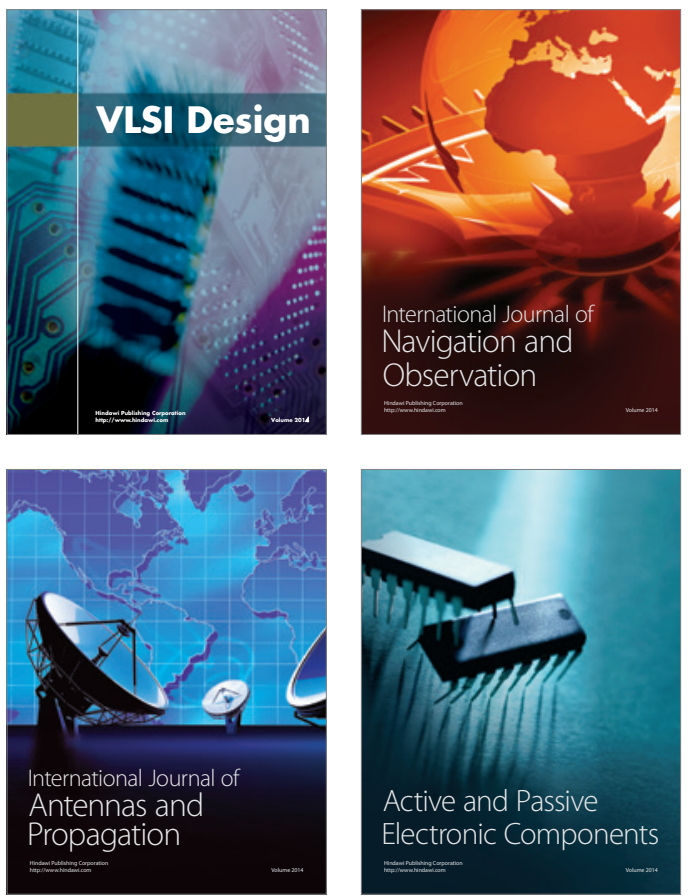
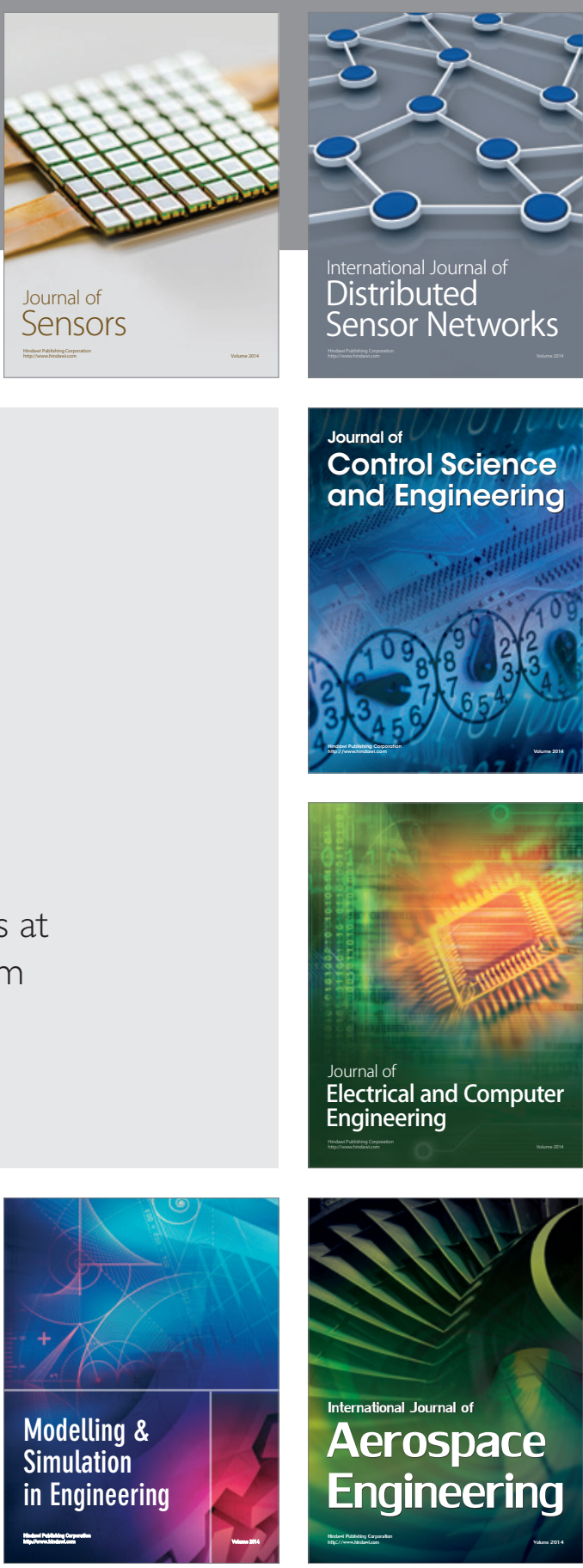

International Journal of

Distributed

Sensor Networks

Journal of

Control Science

and Engineering
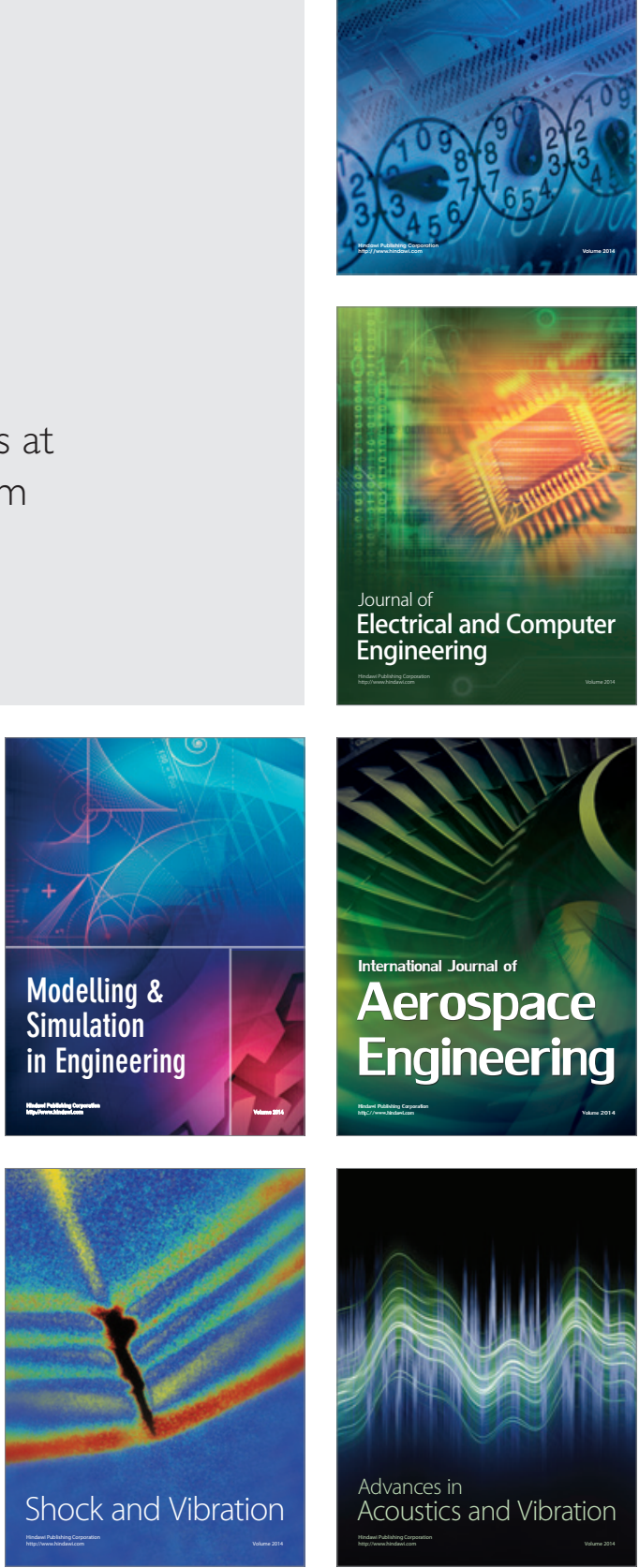